CAHIERS DE

NARRATOLOGIE
Cahiers de Narratologie

Analyse et théorie narratives

$16 \mid 2009$

Images et récits

\title{
Claude Simon : une contestation du texte par l'image
}

Bérénice Bonhomme

\section{CpenEdition}

Journals

Édition électronique

URL : http://journals.openedition.org/narratologie/1025

DOI : 10.4000/narratologie.1025

ISSN : 1765-307X

Éditeur

LIRCES

Référence électronique

Bérénice Bonhomme, «Claude Simon : une contestation du texte par l'image », Cahiers de Narratologie [En ligne], 16 | 2009, mis en ligne le 25 mai 2009, consulté le 30 avril 2019. URL : http:// journals.openedition.org/narratologie/1025; DOI : 10.4000/narratologie.1025

Ce document a été généré automatiquement le 30 avril 2019.

\section{(c) $($ i) $(9)$}

Cahiers de Narratologie - Analyse et théorie narratives est mis à disposition selon les termes de la licence Creative Commons Attribution - Pas d'Utilisation Commerciale - Pas de Modification 4.0 International. 


\title{
Claude Simon : une contestation $\mathrm{du}$ texte par l'image
}

\author{
Bérénice Bonhomme
}

\section{Vers l'image}

1 La preuve de l'importance de l'image dans l'œuvre de Claude Simon n'est plus à faire. Une grande place est ainsi accordée à la peinture, la photographie mais aussi la bande dessinée, la cartographie, le schéma, les graffiti, la publicité, le blason, l'image d'Épinal, le timbre, sans oublier, bien entendu, le cinéma.

Une image absente

2 Le rôle de l'image plastique, sous toutes ses formes, apparaît d'abord comme celui d'un stimulus à l'écriture. On pense évidemment à la place des peintures de Bacon, Dubuffet et Delvaux dans Triptyque. Les images apparaissent comme des générateurs textuels, les descriptions de tableaux s'intégrant parfaitement dans le récit simonien et en constituant des points dynamiques. On connaît aussi l'importance des cartes postales dans l'économie du roman Histoire et le rôle des photographies en particulier dans L'Herbe, Histoire ou L'Acacia. Cette utilisation apparait comme encore plus centrale dans des oeuvres comme Femmes, sur vingt-trois peintures de Joan Miro ou Orion aveugle où le texte est publié avec des images qui sont manifestement à l'origine de l'écriture.

3 Mais à y regarder de plus près, la place de l'image comme fédératrice du texte semble ambiguë. Tout d'abord certaines images ne précèdent pas l'écriture. Ainsi Claude Simon nous apprend que quelques-uns des aspects de l'iconographie de Orion Aveugle se sont ajoutés plus tard, « illustration "après coup" » qui lui semblait s'harmoniser avec ce qu'il avait tenté de faire ${ }^{1}$, et cet ajout a posteriori suppose donc un dialogue plus vaste entre image et écriture dans l'économie créative de cette oeuvre.

4 Notons aussi que Claude Simon a choisi de republier le texte de Femmes, cette fois sans les images, sous le titre de La Chevelure de Bérénice. Pascaline Mourier-Casile parle à ce sujet de « mise hors-jeu, hors-texte des images » :

par le retranchement radical des images et le choix d'un nouveau titre, La Chevelure

de Bérénice, (pourtant identique textuellement à Femmes), est devenu un autre texte 
qui demande à être lu autrement. Toute mention de ses stimulii picturaux risquerait d'en fausser la lecture ${ }^{2}$.

De même, le texte de d'Orion Aveugle se retrouve dans Les Corps conducteurs, sans les images, et Claude Simon déclare à son sujet: "si Les Corps conducteurs devait être accompagné de l'image, de ses stimulants extra-textuels, alors ce serait un échec pour $\mathrm{moi}^{3}$ ». L'image est mise à distance, volontairement absentée. En fait, les relations entre le texte simonien et l'image sont construites autour de ce paradoxe, celui d'un dialogue en creux, d'un va-et-vient perpétuel qui a pour dynamique l'absence. On peut d'ailleurs remarquer, dans les romans, l'obsédante thématique de la trace, de l'image dont il ne reste que l'empreinte, comme les formes de cadres désormais absents restant inscrites sur les murs du Palace. Parler d'images dans les romans de Claude Simon est une " image » puisque, sauf exception, elles ne sont pas présentes concrètement. Cette relation in absentia permet de rendre l'image la plus "plastique" possible, dans le sens de "susceptible de transformations, de modulations", et pouvant même s'animer (dans le cas d'images fixes) si le texte le requiert. Cette image absente peut d'ailleurs être une invention de l'auteur, comme le dessin décrit au début des Géorgiques, « dessin imaginaire ${ }^{4}$ » qui semble pourtant bien réel au lecteur. Plus subtil est le cas de la photographie de l'atelier qui est au cœur d'Histoire, photographie dont,

la lumière terne de l'hiver ayant probablement nécessité un temps de pose assez long de sorte que pendant les quelques secondes durant lesquelles l'obturateur est resté ouvert sa tête a occupé successivement trois positions : la première (où elle apparaît de profil) transparente et très pâle, les deux autres (de trois quarts cette fois, et regardant l'appareil) légèrement à droite et en arrière du profil, les trois images se chevauchant et reliées par des traînées, des stries parallèles, comme si l'on avait appuyé le doigt sur la première encore fraîche pour la faire glisser ${ }^{5}[. .$.$] .$

6 Cette photographie, qui existe bel et bien, est reproduite dans le livre de Lucien Dällenbach. Mais, ce qui saute d'emblée aux yeux, c'est précisément l'absence de tout bougé, de toute multiplication du visage du peintre, élément pourtant essentiel de la description scripturale. Manifestement, Lucien Dällenbach a été intrigué par cette absence et a recherché une photographie de l'atelier plus conforme à la description, jusqu'à ce que Claude Simon avoue dans une lettre de 1986 : « il n'existe pas d'autre photo de l'atelier que celle que tu connais. Celle «bougée » est inventée de toutes pièces ${ }^{6}$ ». Si l'image est un " générateur textuel », l'inverse est également vrai et l'image est créée ou recréée par le biais de l'écriture. C'est le cas, par exemple, lorsque l'image est présente comme dans Photographies ou Album d'un Amateur. Dans Photographies, les quelques mots de légende influent sur la photographie, lui insufflant une temporalité, comme dans Jeux et Plus tard ${ }^{7}$, ou encore une portée métaphorique comme Jambes qui donne à voir un arbre ${ }^{8}$. Dans Album d'un Amateur, le texte est davantage présent. Ainsi, dans la photographie des trois jeunes danseuses, le texte descriptif accompagne avec minutie l'image, va-et-vient envoûtant qui apprend à regarder au plus près, la «torsion hélicoïdale d'une jupe » ou «le pantalon serré aux chevilles par les pinces ${ }^{9}$ ».

7 Le choix de l'absence d'image dans les romans participe d'une véritable mise en tension du lecteur qui ressemble au mécanisme voyeuriste. Ces images absentes sont entêtantes au point que le lecteur va partir en quête pour tenter de les mettre enfin face au texte. Ainsi Lucien Dällenbach rapproche des photographies prises par Claude Simon de bribes de texte de La Bataille de Pharsale ${ }^{10}$. D'une certaine façon, le lecteur simonien se met à imiter la recherche des origines initiée par le narrateur simonien qui scrute jusqu'à l'hallucination ses photographies de famille. Cette tension apparaît vite comme vaine et 
se heurte à des obstacles insurmontables (comme la photographie inventée). Mais c'est un battement d'aile du papillon qui provoque un cyclone, entraînant une dynamique irrépressible, une image en signalant une autre. Un dialogue commence alors entre les différents romans et l'œuvre photographique et cela par le biais des images. Ainsi les branches de l'acacia constituent un motif récurent que l'on retrouve de roman en roman jusqu'à ce qu'on les voie photographiées sur la couverture du roman L'Acacia. Les descriptions du changement de la garde devant le tombeau de Lénine dans Le Jardin des Plantes sont présentes dans le recueil de photographies Album d'un Amateur, le phallus de Délos décrit dans La Bataille de Pharsale se retrouve lui aussi dans Album d'un Amateur, la boutique " aux trois nègres " qui est cité dans Les Géorgiques ${ }^{11}$ existe en photo (catalogue de l'exposition Claude Simon à la galerie municipale du château d'eau en 1992)... Se met en place un réseau d'images et de mots, profondément entrelacés, mot et image devenant deux faces d'un même objet.

Mots et images

Selon Yves Peyré, «on peut exprimer deux propositions parfaitement contradictoires Claude Simon est avant tout un homme de l'image et il est plus que tout un homme des mots. Les deux sont "vrais"12" ", formulation paradoxale certes, mais qui a le mérite d'exprimer avec évidence un des aspects les plus fascinants de l'œuvre simonienne où les images sont dans les mots et les mots dans les images.

9 Tout d'abord, de façon récurrente, Claude Simon transforme la parole pour la rendre visuelle. Ainsi dans L'Herbe :

la fumée se détachant de ses lèvres, les paroles se détachant de ses lèvres, restant comme la fumée un instant suspendues au-devant de ses lèvres, une boule grise roulant sur elle-même, les sons prononcés, les mots roulant les uns sur les autres, c'est-à-dire montrant leurs diverses faces, leurs diverses combinaisons - ce pourquoi on dit sans doute «tourner et retourner des paroles »- puis (les mots, l'assemblage de mots) s'effilochant, se désagrégeant, se dissolvant dans l'air nocturne ${ }^{13}[. .$.$] .$

10 Notons que c'est aussi l'occasion de prendre au pied de la lettre une expression idiomatique «tourner et retourner des paroles dans sa bouche » et de l'imager. On pense ici à la bande dessinée et aux bulles qui sortent de la bouche des personnages, intuition confirmée par la suite dans Histoire :

comme si je pouvais positivement le voir ravaler les mots sur le point de sortir, comme si je pouvais même les voir se former à la façon de ces bulles qui se gonflent entre les lèvres des personnages des bandes dessinées ${ }^{14}[. .$.

11 Forme intégrée de récit en image, la bande dessinée combine dessin et texte. Elle constitue donc un support parfait de cette «tension rentrée vers le visible » qui travaille le texte simonien. Les paroles se matérialisent et sont dès lors à voir et non plus à entendre :

regardant en réalité un spectacle intérieur, peut-être la forme la couleur des mots qu'elle vient de dire comme s'ils lui apparaissaient non pas imprimés et enfermés dans des bulles mais surgissant du néant l'un après l'autre avec leurs sinuosités leurs barres leurs verticales leurs méandres leurs ondulations leurs coupures abruptes se complétant se reliant grossissant puis s'immobilisant restant là suspendus dans l'air lui aussi immobile continuant à vibrer silencieusement redoutables énigmatiques chargés de sens multiples jusqu'à ce que la phrase la réplique suivante les repousse s'installe à leur place (à la manière de ces images des lanternes magiques glissant de droite à gauche puis de gauche à droite, l'une chassant l'autre, chacune immobile un moment pendant que l'opérateur cherche à tâtons dans l'obscurité la plaque suivante, l'installe, et la fait brusquement coulisser 15) $[\ldots]$. 

«barres ", leurs « verticales ", le problème de la signification passant au second plan. La conversation se transforme en spectacle de lanterne magique, un des ancêtres du cinéma, ce qui accentue le caractère proprement visuel, "vibrant silencieusement " de cette parole. En outre, dans Triptyque, la voix-off d'un des films projetés est figurée comme image mouvante, tournant lentement sur elle-même en lettres lumineuses, tout comme les éléments architecturaux filmés pivotaient «avec majesté, présentés sur un plateau tournant, à la façon de ces chefs-d'œuvre de pâtisserie pompeusement exposés dans la vitrine d'un confiseur ", image et parole se trouvant alors assimilées :

[...] la voix monumentale du commentateur se faisant en même temps plus pompeuse tandis que les mots qu'il articule (milliardaires, fastueux, rois, palace, luxe, splendide) semblent pour ainsi dire tourner lentement eux aussi, comme s'ils défilaient en lettres de feu sur ces bandeaux publicitaires aux angles des immeubles [...] traînant derrière eux la rétine éblouie des crinières d'aigrettes lumineuses ${ }^{16}$.

Le champ lexical attaché à la voix-off est péjoratif (" pompeuse »), alors qu'après la transformation des paroles en images, ce champ devient nettement plus positif et dénote la fascination de l'auteur pour le langage lumineux du cinéma (« rétines éblouies ») qui l'entraîne vers la poésie (« crinières d'aigrette lumineuses »). Mais cette matérialisation des paroles n'est pas sans risque et porte en elle sa destruction : comme le résidu, les indestructibles décombres de ces villes anéanties par quelque séisme, l'éruption d'un volcan, la pluie de feu, et où les cadavres des couples enlacés subsistent intacts, momifiés, ardents, insoucieux, juvéniles et priapiques ${ }^{17}$ [...].

les paroles deviennent visibles, les mots écrits eux aussi sont gros de leur pouvoir d'image. Ainsi les lettres n'ont pas renoncé à la puissance du dessin et évoquent des illustrations de contes de fées :

l'H démesuré, emphatique, en forme de deux parenthèses se tournant le dos et reliées par un trait onduleux, les extrémités des parenthèses s'enroulant en colimaçon comme les motifs de ces grilles rongées de rouille qui gardent encore l'entrée des parcs envahis par les ronces ${ }^{18}[\ldots]$.

16 La signification des mots tient autant à leur aspect visuel qu'au code du langage :

avec ce B majuscule au double renflement opulent et majestueux initiale aussi de Barcelone comme un poitrail de pigeon imposante et orgueilleuse géante fardée de bleu de blanc gras en robe safran ${ }^{19}[\ldots]$.

17 Ainsi ce travail sur les lettres transforme-t-il les mots en images comme ce « elle aCCourt aile de ses cheveux défaits figure volant les deux boucles jumelles » :

les deux $\mathrm{C}$ semblables à deux dos ou plutôt au même dos répété deux fois comme les contours d'un personnage sur une photo bougée forme fuligineuse d'un corps ou plutôt d'un mouvement une jambe levée semblable à quelque figure volante écartelée sa fente la moule ce coquillage au goût de $\operatorname{sel}^{20}[. .$.$] .$

C'est la matérialité du mot, son dessin qui devient le support de l'imaginaire. Les mots constituent l'image-trace d'une absence, comme l'est l'empreinte photographique. Cette perception se rapproche de celle que l'on peut avoir dans l'enfance lorsque la frontière entre mot et image est beaucoup plus poreuse et où l'apprentissage de la lecture s'appuie sur des supports imagés. D'ailleurs Claude Simon évoque «ces rebus où un objet, une lettre majuscule se déplace sur une paire de jambes en fil de fer (canne va, A court ${ }^{21}$ )» ancrant délibérément la problématique de ces mots-images dans le monde de l'enfance. Jeu d'enfance qui fait accoucher les mots d'images et où l'on retrouve logiquement des 
références au monde enfantin et plus précisément au soldat de plomb qui hante les romans simoniens :

fracas aussi dans Frascati Et alors sans doute à cause de la consonance italienne du mot l'image stéréotypée non de soldats en uniformes de la dernière guerre mais les silhouettes serties de plomb d'officiers sabre au clair et de turcos ${ }^{22}[\ldots]$.

19 On sait, qu'en partie, l'ontogenèse mime la phylogenèse. En rattachant le mot à l'image, Claude Simon lui redonne sa force archaïque de mythogramme, objet biface d'imageparole. Ainsi le mot possède une puissance magique incantatoire : «le nom du monastère [...] comme un éclair noir et or zébrant les ténèbres : Zagorsk ${ }^{23} »$. Notons d'ailleurs les évocations récurrentes de la préhistoire et des dessins retrouvés dans les cavernes. Dans un même ordre d'idées, on peut également signaler la fascination de Claude Simon pour les graffiti qu'il décrit et photographie à de nombreuses reprises. La plupart du temps, ces fresques, entre écriture et image, permettent d'atteindre aux fondamentaux archaïques de l'humain, c'est-à-dire la mort et la sexualité :

ma pine enfoncée en elle moi étendu rigide mort pouvant me voir sous la forme de ce guerrier ou chasseur ithyphallique de la préhistoire réduit à quelques traits charbonneux comme si j'étais fait de barres raidi tout mon corps aux membres durcis noirs mes os parallèles qu'on retrouverait des siècles plus tard dessinés sur les parois des cavernes ${ }^{24}[\ldots$ ].

21 La question de la sexualité ou de la mort se devine sous plusieurs de ces mots-images, « aCCourt » évoque la « fente, la moule »,

nom (Memel) qui faisait penser à Mamelle avec dans son aspect je ne sais quoi (les deux e blanc peut-être) de glacé ville noire couronnée de neige auprès d'une mer gelée livide habitée par les femmes slaves aux cheveux de lin aux seins lourds (les deux 1 de mamelle suggérant la vision de formes jumeLLes se balançant) et neigeux ${ }^{25}[\ldots .$.$] ,$

de même le trajet de la guerre, « l'immémorial itinéraire [est] jalonné de noms semblables à des armures, crénelés, armoriés, aux senteurs de fer, de poudre, de camps ${ }^{26}[. .]$.$» .$

23 Ainsi Claude Simon suscite-t-il dans les mots des images. Il est donc normal que la manière de les écrire, la calligraphie, soit longuement évoquée dans l'œuvre. C'est particulièrement sensible dans Les Géorgiques où l'auteur travaille à partir d'archives familiales et rend compte de la réalité manuscrite des écrits :

L'écriture impulsive, fougueuse, l'encre pâle, transparente, d'un brun rouille presque rose : avec leurs festons irréguliers de jambages, d'entrelacs, de ratures, d'arabesques, les lignes dessinent sur les feuillets jaunis comme de minces bandes de dentelles déchiquetées et fanées ${ }^{27}$.

24 L'aspect purement visuel de l'écriture est mis en lumière, couleur de l'encre, entrelacs, lignes, la page d'écriture est décrite comme un tableau. Le sens même des mots se perd:

Puis regardant sans parvenir à en pénétrer le sens, les lignes, les noms d'aliments tracés d'une encre amarante et délavée, ou plutôt sans parvenir à ce que les rangées de jambages, de boucles et de hampes carminées coïncident, s'identifient avec comment est-ce déjà ${ }^{28}[. .$.$] .$

25 Entre les arabesques des mots, des images naissent « comme la crinière d'un cheval au galop flottant dans le vent ${ }^{29}$ » créées par l'encre encore fraîche balayée par la main. Ainsi, dans la liste des chevaux faite par le général :

trois ou quatre lignes que les biffures semblaient envelopper de spirales, comme

d'épineux faisceaux d'ossements entortillés de rouille, de bandelettes bitumeuses: 
des momies, les squelettes de ces coursiers chargés d'accompagner dans l'au-delà quelque pharaon ${ }^{30}[. .$.$] .$

Palace. Dans ce roman, l'Italien éprouve le besoin de dessiner un schéma pour expliquer à l'étudiant comment s'est déroulé l'attentat dont il est l'auteur. Son schéma indique la place des objets et le déplacement des personnages, démarche qui évoque très fortement les exercices de mises en scène que propose Eisenstein à ses étudiants et les schémas qui en découlent ${ }^{33}$. L'Italien construit peu à peu sur la feuille un espace :

en train de dessiner, [...] vers l'intérieur du rectangle, une courte ligne ondulée, comme un serpent, une sinusoïde, puis, sans explication, au-dessus de celle-ci et à la verticale [...] un cercle ou plutôt plusieurs cercles [...] concentriques, se superposant, ou légèrement excentriques les uns par rapport aux autres, découvrant lorsqu'il écarta la main, quelque chose qui ressemblait à une pelote de laine $^{34}[. .$.$] .$

31 Mais à force d'accumuler traits et lignes, le dessin devient si complexe qu'il en est indéchiffrable. Ni le narrateur ni le lecteur ne le comprennent plus. Ici, par opposition à Eisenstein qui se servait du schéma pour communiquer plus clairement, Claude Simon le complexifie jusqu'à ce qu'il devienne incompréhensible. On pourrait objecter que le schéma est difficile à comprendre parce qu'il n'existe pas en tant que tel dans le roman mais qu'il n'est présent qu'en mots. Pourtant, si l'on reporte fidèlement les indications du texte et que l'on en construit le plan, le résultat n'est pas plus convaincant. Le schéma 
n'est alors plus seulement un schéma. Il donne à voir autre chose, devient une image à part entière, s'écartant du référent premier pour donner à voir des «serpents » et des " pelotes de laine ». Ce processus se retrouve dans La Route des Flandres dans la description du champ de manoeuvre :

de sorte que le trajet suivi par chaque unité aurait pu être schématiquement représenté par une de ces lignes fléchées ou vecteur [...] les collines figurées sur la carte au moyen de petits traits en éventail bordant la ligne onduleuse d'une crête, de sorte que le champ de bataille semble parcouru de mille-pattes sinueux, chaque corps de troupe étant représenté par un petit rectangle à partir duquel s'élance le vecteur correspondant, chacun d'eux se recourbant en l'occurrence de façon à affecter à peu près la forme d'un hameçon ${ }^{35}[. .$.$] .$

L'activité configurante du regard ne semble plus pouvoir s'arrêter, donnant sans cesse à voir autre chose, le schéma apparaissant comme un des lieux privilégiés du feuilletage cher à l'auteur. L'on peut évoquer aussi la carte de la ville dans Le Palace. Le plan devient quadrillage régulier puis grille d'égout sous laquelle se cache une énigme, l'énigme de la révolution tout aussi bien que celle de la construction du livre. De même, un plan dans Les Corps Conducteurs perd contact avec son référent pour devenir mur ou damier :

Sur le plan de la ville les blocs des maisons et des gratte-ciel dessinent des rectangles légèrement grisés, de tailles et de formes diverses, quelquefois étirés en hauteur, d'autres fois presque carrés, selon les intervalles plus ou moins grands entre les rues et les avenues parallèles qui se coupent toutes à angle droit. L'ensemble prend l'aspect d'un mur aux joints apparents de ciment blanc, ou encore d'un vaste damier aux cases irrégulières, tachées parfois de rouge aux emplacements des gares, des musées et des principaux monuments publics ${ }^{36}$.

Si l'œuvre simonienne n'est pas avare en cartographies, elle est conjointement table d'orientation et de désorientation, et aboutit à la multiplication des schémas qui sont là comme autant de pelures d'images supplémentaires. Il existe un véritable plaisir de la carte, motif récurent :

De fines lignes carmin dessinent des triangles, des parallélogrammes, convergent en certains points, s'écartent de nouveaux, s'entrecroisent au-dessus d'océans bleu pâle, d'étendues vertes ou jaunâtres ${ }^{37}[. .$.$] .$

mais c'est une carte-image qui vaut pour elle-même, objet autonome qui ne renvoie à aucun référent et qui n'est pas là pour apporter une quelconque clarification. Il n'est que de voir les nombreuses cartes imaginaires que le regard suscite partout où il se pose :

à un endroit l'humidité [...] attaquant peu à peu le métal à partir d'une simple éraflure...[...]...avait gagné de proche en proche finissant par l'élargir au point qu'elle ressemblait maintenant à une île de forme allongée, la Crète par exemple ${ }^{38}$ [...].

Notons par ailleurs que Claude Simon a fait de la structure de ses différents romans des représentations graphiques (par exemple le trèfle pour La Route des Flandres). Ces représentations semblent davantage correspondre à la fonction première d'un schéma et offrent une vision concentrée de la composition des romans. Cependant deux remarques doivent être faites. Tout d'abord ces schémas n'ont pas uniquement un rôle épistémique mais peuvent également jouer le rôle de blason des romans, ainsi le trèfle de La Route des Flandres (qui reste le dessin le plus connu) ou le « tracé sinueux » que l'on retrouve dans la préface d'Orion aveugle ou en quatrième de couverture d'Album d'un amateur. D'autre part, dans ces schémas, on peut remarquer la prédominance du « tracé sinueux » qui se répète, toujours semblable et toujours différent, les extrémités se rejoignant ou pas, les points de contact évoluant. Ce tracé sinueux est, comme son nom l'indique, bien difficile à suivre et 
s'il nous renseigne sur la structure du roman simonien qui s'organise autour de quelques motifs obsédants, de schéma en schéma, les nœuds du réseau sont de plus en plus difficiles à repérer et ce "tracé sinueux " évolue vers une certaine opacité qui nous renvoie encore une fois au trait lui-même et à la sensation brute de l'image.

Si pour Claude Simon le texte tend vers l'image jusqu'à faire d'une représentation graphique le blason de nombre de ses romans, l'image, inversement, apparaît formée à partir d'éléments constitutifs de l'écriture. Ce renversement semble évident lorsqu'on voit la photographi ${ }^{39}$ représentant un mur et intitulé « Page d'écriture ». Soulignons aussi le rôle essentiel que joue le $\mathbf{V}$ dans la construction visuelle du monde simonien. Ce $\mathbf{V}$ que l'on retrouve dans un nombre incalculable de descriptions ( "plastron en $\mathrm{V}^{40}$ », « les jambes écartées en $\mathrm{V}^{41} », " \mathrm{~V}$ du corsage ${ }^{42}$ ), suivi de près par le $\mathrm{S}$ ( $\mathrm{S}$ couché et irrégulier ${ }^{43}$ ", " le contour inférieur du ventre dessine un $S$ oblique $\left.{ }^{44} »\right)$, raccroche à chaque instant ce qui est vu à ce qui s'écrit, véritable pont entre mot et image.

En fait, tout ce qui est vu tend à devoir être lu :

et tout à coup pourtant il le vit (le parapet ennemi), très haut, lui parut-il, au-dessus d'eux, et noir sur noir (comme des lignes d'écriture épineuses, menaçantes, tracées sur la nuit), les barbelés ${ }^{45}[. .$.$] .$

Les personnages sont ramenés à leur statut d'écrit comme le personnage de Triptyque comparé à une « tache d'encre ${ }^{46}$ » ou le peintre d'Histoire :

le Hollandais juché dans une pose simiesque [...] sur son haut tabouret, comme s'il était peint tout entier d'une seule couleur (noir ou bleu de Prusse), lui, sa casquette de marinier, ses pantoufles à carreaux, et, en face, son chevalet, le tout semblable à quelque idéogramme oriental: deux signes, deux caractères tracés l'un à côté de l'autre en quelques coups de pinceau ${ }^{47}[. .$.$] .$

39 Le personnage de Triptyque est mal éclairé (donc sombre) et celui d'Histoire est vu en contre jour, c'est donc grâce à une caractéristique propre à l'image, la lumière, que le visuel tend vers l'écrit. Cet entre-deux est encore affirmé par le terme d'idéogramme. L'idéogramme est un symbole graphique représentant un mot ou une idée. Ce n'est certes pas un pictogramme, mais il a des caractéristiques graphiques (d'autant plus apparentes pour un public occidental n'en comprenant pas la signification). Il n'est pas indifférent également que ce que représentent ces "deux signes», ce soit un peintre, donc un homme d'images. Dans l'œuvre de Claude Simon, ce qui constitue l'écriture est considéré autant à voir qu'à lire.

De même dans les descriptions des images, le champ lexical de la ponctuation est particulièrement obsédant :

Deux parenthèses couchées, comme deux cupules, soulignant chacune un point, figurent les seins. Au-dessous de la sirène clapotent trois courtes rangées d'accents circonflexes alignés comme des tentes d'un camp militaire ou les crêtes pointues d'une eau agitée ${ }^{48}$.

41 C'est le cas également lors des perceptions visuelles directes comme le bec de pigeon « en forme de virgule ${ }^{49}$ " et «les bords des paupières fermées dessinent deux minces croissants, comme des parenthèses horizontales, au-dessus des joues fraîches ${ }^{50}$ ». La prégnance des termes indiquant des procédés de ponctuation pour décrire tout ce qui est visuel doit nous faire réévaluer l'aspect purement graphique de l'utilisation de cette ponctuation dans le texte même de l'œuvre. Ainsi la construction de la phrase simonienne se découvre sous un aspect purement graphique où les parenthèses valent aussi pour leur forme. 
L'image comme ferment contestataire du livre vers un statut de reproduction. Ce n'est pas seulement le mot qui est repris, mais son apparence graphique. C'est encore plus évident dans la description d'une page de programme de cinéma dans Les Corps conducteurs :

Au-dessus de sa tête est écrit en cursive le mot Simultanéo encadré de deux points d'exclamation, puis, en capitales: HOY-ALAMEDA, MINERVA.DESDE LAS 13 HRS.! TRIPLE COLOSAL !, et dans un cartouche noir : LA VENUS MALDITA Eastmancolor, * MAYORES DE 21 ANOS $^{53}$.

Il y a un véritable va-et-vient entre reproduction et description. Ainsi le mot «simultanéo » est typographié dans une police particulière. En revanche, son encadrement par deux points d'exclamation est exprimé par le texte, mais non effectif. Cette tentation analogique va trouver son point culminant dans La Bataille de Pharsale roman qui propose une réflexion sur les signes et où se multiplient les symboles graphiques et des «comment 
dire: idéogrammes ${ }^{54}$ ?». Ainsi on voit une enseigne de boutique de vêtement jouant sur les lettres et les dessins de veston et de pantalon (

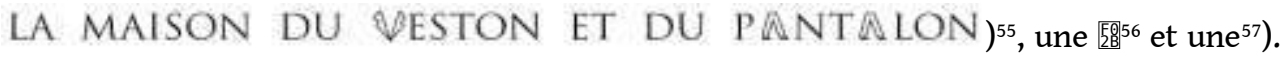
Le soubassement d'images qui sous-tendait le texte troue la surface des mots et modifie radicalement l'aspect du texte. Là encore cela a à voir avec un fond archaïque :

je voudrais une

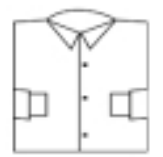
présages sacrifice aux dieux pour se concilier savoir... Entrailles questionnées fumant tièdes sur la pierre de l'autel ${ }^{58}[. .$.$] .$

Avec la résurgence de l'image, sur le devant de la scène apparaît la magie.

La mise en page également propose un aspect graphique du texte. C'est évident dans Le Jardin des Plantes qui est particulièrement travaillé à cet égard. Les particularités typographiques des premières pages reproduiraient la disposition des notes préparatoires du roman. En effet, même si en cours d'élaboration cette disposition typographique à subi de nombreuses modifications, il en est resté « l'idée ${ }^{59}$ ». On a vu le caractère plastique des manuscrits de Claude Simon, c'est donc une idée-image qui a organisé la structure et la mise en page de tout le début du Jardin des Plantes. En outre, Claude Simon affirme « cette disposition palliait dans une certaine mesure l'impossibilité où l'on est de dire en même temps des choses qui sont pourtant saisies ensemble, puisque l'écriture n'a qu'une dimension, la linéarité ${ }^{60} \%$. C'est vers la simultanéité, qualité que Claude Simon associe à l'image (en opposition avec la littérature), que tend ici le texte. En voulant s'approcher d'une caractéristique propre à l'image, le texte se modifie en profondeur, prenant luimême un aspect plastique.

D'autre part, le cinéma et ses techniques transforment la manière d'écrire de l'écrivain. Le cinéma a pour effet de promouvoir l'analogie comme ressort essentiel de son double niveau de fonctionnement : ressemblance des images avec le monde et ressemblance des images entre elles. Or Claude Simon déclare: «le roman doit être un jeu de miroirs internes ${ }^{61}$ ». L'écriture avance de mot en mot mais aussi d'image en image, se construisant à travers tout un réseau d'échos. Toute l'œuvre est jalonnée de renvois, de reports de texte, de scènes récurrentes, de motifs repris. Une des caractéristiques les plus spectaculaires de l'œuvre de Claude Simon est ce retour d'images que l'écriture remet sur le métier pour, sans cesse, les retravailler. Chaque élément contient sa pelure d'images comme ces hommes politiques:

ils remplissent à demi le vaste hémicycle où dans les stalles inoccupées semblent toujours siéger, bismarckiens, gourmés et réprobateurs, les fantômes de messieurs à favoris comme ceux dont on voit les portraits à l'intérieur des couvercle des boîtes à cigares ${ }^{62}[. .$.$] .$

Ce soubassement constant d'images finit par faire écran. Le romancier nous soumet ici, non à une suspension de l'illusion référentielle, mais à une surenchère si complexe de cette illusion qu'il finit par en noyer la fonctionnalité. Ou plutôt le visuel ainsi démultiplié par les reflets et les images ne peut servir de caution d'authenticité de ce qui est vu, mais au contraire, en mélangeant aussi inextricablement réalité supposée et fiction, c'est l'existence même d'une réalité derrière les images qui est mise en doute. Ce que l'on voit dans les stalles inoccupées, ce sont des fantômes. De même le général des Géorgiques finit par confondre la carte de son domaine avec le domaine lui-même : 
51 il n'avait fait que traverser sans s'arrêter ce paysages, ces champs, ces collines, ce vallon, ces halliers qui pendant toutes ces années n'avaient existé pour lui que comme des choses pas tout à fait réelles, sans autre matière que l'immatérielle mémoire, abstraitement figurées sur ce plan qu'il emportait partout avec lui [...] usant non de charrues ou de herses mais de cette encre brune, couleur rouille ${ }^{63}[. .$.$] .$

52 L'image joue un rôle de mise à distance. Ainsi de l'importance de l'empreinte photographique dans l'œuvre, image trace de ce qui a été mais qui n'est plus, séparation d'avec les choses par l'intermédiaire de l'écran du temps. Plus généralement, la thématique de l'empreinte est liée à celle de l'absence :

rien ne bougeant immobile comme si mon corps étendu s'enfonçait lentement laissant son contour dessiné sur le drap le matelas et à la fin il ne resterait plus que ce trou ayant vaguement la forme d'un homme comme ces silhouettes en bois découpé que l'on dresse pour servir de cibles au $\operatorname{tir}^{64}[. .$.$] .$

A la fin il ne reste plus que des images, ou plutôt la trace d'une image.

L'existence même d'images créées par Claude Simon comme ses photographies et son court-métrage modifie le texte écrit. Lorsque Claude Simon adapte Triptyque au cinéma sous la forme d'un court-métrage intitulé L'Impasse, de nombreux épisodes et personnages disparaissent. Le montage montre avec évidence que c'est un homme âgé qui repense aux différentes étapes de sa vie: l'enfance, le mariage et la vieillesse. Ce lien apparaît beaucoup plus évident dans le film que dans le livre. La trame a été simplifiée, ne laissant que trois moments qui pourraient s'échelonner dans le temps. C'est ainsi que le film souligne un sentiment nostalgique qui éclaire d'un jour nouveau le livre.

Ainsi l'image apparait-elle comme une tension en creux de l'écriture simonienne. Touchant aux forces archaïques et magiques, l'image modifie le texte de l'intérieur, lui rendant sa part d'enfance et de magie. Elle joue également un rôle essentiel dans la dynamique de relations du texte au référent. Elle le modifie enfin de l'extérieur en métamorphosant notre regard par la force de contestation d'un récit qui permet de proposer de voir autre chose.

\section{NOTES}

1. «Attaques et stimuli », entretien entre Claude Simon et Lucien Dällenbach, in Claude Simon, Lucien Dällenbach, Seuil, 1988, p. 180.

2. « Je(ux) d'images : Simon au miroir de Miro », Pascaline Mourier-Casile in Les Images chez Claude Simon, des mots pour le voir, dir. Stéphane Bikialo et Catherine Rannoux, Revue La Licorne, 2004, p. 193.

3. «La fiction mot à mot». Communication, suivie d'une discussion, au Colloque de Cerisy, 20-30 juillet 1971. Le Nouveau roman : Hier, aujourd'hui, tome II : Pratiques, U.G.E., 1972, p. 73-97. et p. 99-116. [discussion], p. 110. 
4. «Attaques et stimuli », op. cit., p. 172.

5. Claude Simon, Histoire, Minuit, 1967, p. 267-268.

6. Lettres du 2 octobre 1986 in Claude Simon, Lucien Dällenbach, op. cit., p. 167.

7. Claude Simon, Photographies, Maeght, 1992, p. 34 et 35.

8. Ibid., p. 120.

9. Claude Simon, Album d'un amateur, Rolandseck : Editions Rommerskirchen (Signatur), 1988, p. 8. Voir l'analyse de Ralph Sarkonak, Les trajets de l'écriture, Claude Simon, Paratexte, 1994, p. 133.

10. Lucien Dällenbach, Claude Simon, op. cit., p. 83 et 92 .

11. Claude Simon, Les Géorgiques, Minuit, 1981, p. 207.

12. Yves Peyré, entretien avec Stéphane Bikialo, «Claude Simon, une tension rentrée vers le visible » in Les Images chez Claude Simon, des mots pour le voir, op. cit., p. 83.

13. Claude Simon, L'Herbe, Minuit (Double), 1986, p. 111.

14. Claude Simon, Histoire, op.cit., p. 77.

15. Claude Simon, La Bataille de Pharsale, Minuit, 1969, p. 68.

16. Claude Simon, Triptyque, Minuit, 1973, p. 35-36.

17. Claude Simon, Histoire, op.cit., p. 110.

18. Claude Simon, La Route des Flandres, Minuit (Double), 1984, p. 78.

19. Claude Simon, Histoire, op. cit., p. 168.

20. Ibid., p. 124.

21. Ibid., p. 165.

22. Ibid., p. 336.

23. Claude Simon, L'Invitation, Minuit, 1987, p. 86.

24. Claude Simon, Histoire, op.cit., p. 125-126.

25. Ibid., p. 213.

26. Claude Simon, Les Géorgiques, op.cit., p. 249.

27.Ibid., p. 58.

28. Claude Simon, Histoire, op. cit., p. 138.

29. Claude Simon, Les Géorgiques, op.cit., p. 71.

30. Ibid., p. 368.

31. Yves Peyré et Stéphane Bikialo, op. cit., p 94.

32. Claude Simon, La Bataille de Pharsale, op. cit., p. 260.

33. Eisenstein, Leçon de mise en scène, La Femis, 1989.

34. Claude Simon, Le Palace, Minuit, 1962, p. 61.

35. Claude Simon, La Route des Flandres, op.cit., p. 280-281.

36. Claude Simon, Les Corps Conducteurs, Minuit, 1971, p. 152.

37. Ibid., p. 49.

38. Claude Simon, La Bataille de Pharsale, op. cit., p. 25.

39. Claude Simon, Photographies, op. cit., p. 86.

40. Claude Simon, Le Palace, op. cit., p. 70.

41. Claude Simon, Leçon de choses, Minuit, 1975, p. 34.

42. Claude Simon, Histoire, op. cit., p.145.

43. Claude Simon, Le Palace, op. cit., p. 68.

44. Claude Simon, Histoire, op. cit., p. 271.

45. Claude Simon, Les Géorgiques, op. cit., p. 288.

46. Claude Simon, Triptyque, op. cit., p. 22.

47. Claude Simon, Histoire, op. cit., p. 275.

48. Claude Simon, Leçon de choses, op. cit., p. 156. 
49. Claude Simon, Le Palace, op. cit., p. 9.

50. Claude Simon, Leçon de choses, op. cit., p. 32.

51. Claude Simon, La Corde Raide, Editions du Sagittaire, 1947, p. 12.

52. Claude Simon, Le Palace, op. cit., p. 179.

53. Claude Simon, Les Corps conducteurs, op. cit., p. 146.

54. Claude Simon, La Bataille de Pharsale, op .cit., p. 21.

55. Ibid., p. 21.

56. Ibid., p. 15.

57. Ibid., p. 14.

58. Ibid., p. 44.

59. Le présent de l'écriture ». Entretien avec Jacques Neefs et Almuth Grésillon, Genesis. Manuscrits, recherche, invention, 13, 1999, p. 121.

60. « Parvenir peu à peu à écrire difficilement », entretien avec Jean-Claude Lebrun, L'Humanité, 13 mars 1998.

61.Claude Simon, « La fiction mot à mot », in Nouveau roman : hier et aujourd'hui, op. cit., p. 108.

62. Claude Simon, Les Corps Conducteurs, op. cit., p. 39.

63. Claude Simon, Les Géorgiques, op. cit., p. 377.

64. Claude Simon, Histoire, op. cit., p. 367.

\section{RÉSUMÉS}

L'image joue un rôle central dans l'œuvre de Claude Simon. Le rôle de l'image plastique apparaît d'abord comme un stimulus, mais c'est une image absentée, en creux, mise hors jeux. Inversement, le mot lui-même devient image, tension rentrée vers le visible qui va jusqu'au rébus et au mythogramme, objet biface d'image-parole. L'image comme en creux de l'œuvre a un rôle proprement contestataire, faisant bouger les lignes et les frontières, transformant en profondeur non seulement le texte simonien mais aussi notre regard sur lui.

The image plays a central role in the work of Claude Simon. The image appears as a stimulus at first, but it is an eclipsed image. It remains only as a footprint which is not in play. Conversely, word itself becomes an image, its tension is turned towards the visible which takes it as far as rebus and mythogramme: the biface object of an image-word. As if in the hollow of the work, the image has a genuinely questioning role, making lines and borders move, transforming in depth not only the simonien text but also ourimpression of it.

\section{INDEX}

Mots-clés : stimulus, schéma, mythogramme, magie, image, graffiti, frontière, contestation, Simon Claude, calligraphie

Index chronologique : XXe siècle 


\section{AUTEUR}

BÉRÉNICE BONHOMME

Université de Nice-Sophia Antipolis, CTEL 